\title{
Cerebral Temperature Dysregulation: MR Thermographic Monitoring in a Nonhuman Primate Study of Acute Ischemic Stroke
}

\author{
(D) S. Dehkharghani, (1DC.C. Fleischer, (DD. Qiu, (DM. Yepes, and (DF. Tong
}

\begin{abstract}
BACKGROUND AND PURPOSE: Cerebral thermoregulation remains poorly understood. Temperature dysregulation is deeply implicated in the potentiation of cerebrovascular ischemia. We present a multiphasic, MR thermographic study in a nonhuman primate model of MCA infarction, hypothesizing detectable brain temperature disturbances and brain-systemic temperature decoupling.
\end{abstract}

MATERIALS AND METHODS: Three Rhesus Macaque nonhuman primates were sourced for 3-phase MR imaging: 1) baseline MR imaging, 2) 7-hour continuous MR imaging following minimally invasive, endovascular MCA stroke induction, and 3) poststroke day 1 MR imaging follow-up. MR thermometry was achieved by multivoxel spectroscopy (semi-localization by adiabatic selective refocusing) by using the proton resonance frequency chemical shift. The relationship of brain and systemic temperatures with time and infarction volumes was characterized by using a mixed-effects model.

RESULTS: Following MCA infarction, progressive cerebral hyperthermia was observed in all 3 subjects, significantly outpacing systemic temperature fluctuations. Highly significant associations were observed for systemic, hemispheric, and global brain temperatures ( $F-$ statistic, $P=.0005$ for all regressions) relative to the time from stroke induction. Significant differences in the relationship between temperature and time following stroke onset were detected when comparing systemic temperatures with ipsilateral ( $P=.007)$, contralateral $(P=.004)$, and infarction core $(P=.003)$ temperatures following multiple-comparisons correction. Significant associations were observed between infarction volumes and both systemic $(P \leq .01)$ and ipsilateral $(P=.04)$ brain temperatures, but not contralateral brain temperature $(P=.08)$.

CONCLUSIONS: Successful physiologic and continuous postischemic cerebral MR thermography was conducted and prescribed in a nonhuman primate infarction model to facilitate translatability. The results confirm hypothesized temperature disturbance and decoupling of physiologic brain-systemic temperature gradients. These findings inform a developing paradigm of brain thermoregulation and the applicability of brain temperature as a neuroimaging biomarker in CNS injury.

ABBREVIATIONS: MRSI = MR spectroscopic imaging; NHP = nonhuman primate; 5 LASER = semi-localization by adiabatic selective refocusing; $\mathrm{t}^{\circ}=$ operative day scanning session following endovascular stroke induction; $\mathrm{t}^{1}=$ postinfarction day $1 ; \mathrm{t}^{-7}=$ baseline MR imaging

B rain thermoregulation remains an enigmatic process reflecting the complex interplay of metabolism and cerebrovascular perfusion. $^{1-5}$ A delicate balance of heat-producing and heatdissipating mechanisms ensures a thermal homeostasis, the disturbance of which, even when minor, may engender catastrophic

Received August 30, 2016; accepted after revision November 6.

From the Departments of Radiology and Imaging Sciences (S.D., D.Q., F.T.) and Neurology (S.D., M.Y.), Emory University Hospital, Atlanta, Georgia; and Department of Biomedical Engineering (C.C.F.), Emory University and Georgia Institute of Technology, Atlanta, Georgia.

This work was supported by the American Society of Neuroradiology Scholar Award in Neuroradiology Research (2012-2013, 2013-2014) and an Emory University Research Committee (2013-2014) grant awarded to the lead author and, in part, by National Center for Research Resources and currently the Office of Research Infrastructure Programs of the National Institutes of Health (P51RR000165, OD P51OD011132). brain injury. ${ }^{1,2,6-8}$ Temperature dysregulation proves particularly deleterious as a potentiator of CNS injury following various background insults such as cerebrovascular ischemia, with progressive ischemic damage of neuronal substrates documented in human and nonhuman brains following even modest temperature elevation. ${ }^{9-16}$

Theoretic simulations and limited in vivo study of human and other mammalian brains have suggested the presence of physiologic brain temperature gradients under partial but not immuta-

Please address correspondence to Seena Dehkharghani, MD, Department of Radiology, Neuroradiology Section, New York University, 660 First Ave, New York, NY 10016; e-mail: Seena.Dehkharghani@NYUMC.org

- Indicates open access to non-subscribers at www.ajnr.org

http://dx.doi.org/10.3174/ajnr.A5059 
ble systemic temperature influences. Specifically, during a systemic febrile response, the normally higher brain temperatures at rest may decouple from rising systemic temperatures in a remarkable but controversial notion of selective brain cooling., ${ }^{3,5,17-24}$ This protective physiologic capacity may be notably lost in the injured brain, with failure to preserve cerebral temperatures amid rising systemic temperatures, weakening a critical adaptive mechanism leading to catastrophic brain hyperthermia. ${ }^{18}$

Efforts to develop a generalizable paradigm of brain temperature regulation have been challenged by: 1) brain systemic temperature gradients, which may vary depending on the approach to systemic thermometry (eg, tympanic, sublingual, intravesicular, rectal, and so forth), and 2) the lack of practical techniques permitting direct brain temperature monitoring during physiologic and pathophysiologic conditions. ${ }^{3,17,18,25-29}$ While historical approaches have been limited to the use of costly, invasive, and highly spatially limited implantable temperature probes, optimization of fully noninvasive MR thermometry was recently reported, both in a cytosolic phantom and in nonhuman primates (NHPs), leveraging temperature-sensitive nuclear magnetic resonance phenomena. ${ }^{30,31}$ Recent advancements, including improved magnetic field shimming and multivoxel acquisition strategies, motivate study in an expanded experimental model to better characterize the magnitude and time course of brain temperature fluctuations in acute ischemic stroke. In this report, we prescribed a multiphasic brain MR thermometry protocol, hypothesizing the presence of predictable brain temperature changes and brain systemic temperature decoupling in a minimally invasive NHP ischemic stroke model.

\section{MATERIALS AND METHODS \\ NHP Selection and Preparation}

Three female Rhesus monkeys (Macaca mulatta), 16, 17, and 18 years of age, were sourced from the Yerkes National Primate Research Center. Experimental protocols were designed specifically to minimize subject discomfort and suffering, with full approval of the Institutional Animal Care and Use Committee. Preprocedural animal care consisted of dedicated veterinary supervision with standardized light-dark cycles and routine diet as elaborated previously. $^{32}$

All baseline imaging sessions, stroke-induction experiments, and postischemic imaging protocols shared a common anesthetic protocol, including 3-5 mg/kg of tiletamine/zolazepam (Telazol) or $10 \mathrm{mg} / \mathrm{kg}$ of ketamine followed by induction of general anesthesia by using $1.0 \%$ isoflurane mixed with $100 \% \mathrm{O}_{2}$. The pulse rate, respiratory rate, pulse oximetry, end-tidal gas, and blood pressure were monitored and maintained in the normal range. The subject was immobilized within a head holder throughout the scanning session, and attempts were made to preserve body temperature at $37.5^{\circ} \mathrm{C}$ by using a circulating warm water blanket for heating and withdrawal when supranormal or febrile temperatures were present. A minimally invasive, irreversible endovascular approach developed internally was used, producing superselective, right-sided M2/M3 branch MCA suture embolism, achieved by manual injection and allowing highly controlled infarction induction. ${ }^{32}$

\section{Imaging Protocol}

All imaging experiments were performed on a $3 \mathrm{~T}$ Tim Trio whole-body system (Siemens, Erlangen, Germany). NHP brain imaging was performed by using a custom, 8-channel phased array transmit-receive coil optimized for NHP brain imaging (Invivo, Gainesville, Florida). All subjects underwent a common experimental protocol including the following: 1) approximately 2-4 hours of baseline MR imaging, 1 week before stroke induction $\left(\mathrm{t}^{-7}\right) ; 2$ ) endovascular stroke induction and immediate, approximately 7-hour continuous MR imaging on the operative day $\left(\mathrm{t}^{0}\right)$; and 3) approximately 2 - to 4 -hour postoperative day $\left(\mathrm{t}^{1}\right) \mathrm{MR}$ imaging to characterize delayed temperature changes and stroke evolution. All 3 imaging sessions included noncontrast T1 MPRAGE for structural characterization and to facilitate postprocessing and spatial coregistration of imaging data (TR $=2400$ $\mathrm{ms}, \mathrm{TE}=3.64 \mathrm{~ms}, \mathrm{FOV}=129 \times 129 \mathrm{~mm}$, flip angle $=8^{\circ}, \mathrm{TI}=$ $950 \mathrm{~ms}$, matrix $=192 \times 192$, section thickness $=0.7 \mathrm{~mm}, 128$ sections, 1 average), T2 (fast spin-echo with $\mathrm{TR}=2500 \mathrm{~ms}, \mathrm{TE}=$ $84 \mathrm{~ms}, \mathrm{FOV}=128 \times 128 \mathrm{~mm}$, matrix $=192 \times 192$, section thickness $=2 \mathrm{~mm}, 1$ average), DWI (single-shot EPI sequence with a generalized autocalibrating partially parallel acquisition factor $=3, \mathrm{TR}=4300 \mathrm{~ms}, \mathrm{TE}=90 \mathrm{~ms}, \mathrm{FOV}=576 \times 576 \mathrm{~mm}$, matrix $=64 \times 64$, section thickness $=1.5 \mathrm{~mm}, 30$ directions with b-value $=0,1000 \mathrm{~s} / \mathrm{mm}^{2}$ ), and 3D time-of-flight MRA (either $\mathrm{TR}=23 \mathrm{~ms}, \mathrm{TE}=4.3 \mathrm{~ms}$, flip angle $=18^{\circ}$, matrix $=384 \times 331$, section thickness $=0.4 \mathrm{~mm}, 1$ average; or $\mathrm{TR}=39 \mathrm{~ms}$, $\mathrm{TE}=7.3$ ms, flip angle $=18^{\circ}$, matrix $=448 \times 448$, section thickness $=1.0$ mm, 1 average).

Preoperative baseline scanning $\left(\mathrm{t}^{-7}\right)$ was performed to identify physiologic spatiotemporal intracerebral temperature gradients by using an optimized proton MR spectroscopy protocol (see below). Following stroke induction, the subjects were immediately stabilized and transferred to the MR imaging suite, where they were prepped for a continuous, approximately 7-hour imaging session. Attempts were made to ensure that the duration between conclusion of stroke induction, stabilization, transfer, and initiation of imaging was kept to $<60$ minutes to mitigate expansion and maturation of cytotoxic injury before MR imaging observation could be initiated. A final scanning session was prescribed on the first poststroke day to document final infarction volumes and to establish brain temperatures within the infarcted and noninfarcted hemispheres of the NHP brain. At the conclusion of the $t^{1}$ scanning session, the subjects were euthanized under general anesthesia by using intravenous pentobarbital, $100 \mathrm{mg}$, and transcardial perfusion with saline solution and $10 \%$ buffered formalin following the approved protocols of our Institutional Animal Care and Use Committee.

All scanning data were exported to a separate workstation for analysis (Mac Pro; Apple, Cupertino, California). DWI infarction volumes (cubic centimeters) were calculated in a semiautomated fashion at all time points and for all subjects by using a third-party DICOM viewer (Osirix 64-bit; http://www.osirix-viewer.com).

\section{MR Thermometry}

The theoretic basis of proton resonance frequency thermometry has been expounded previously. ${ }^{33,34}$ Recent enhancements to brain MR thermometry using the temperature-sensitive, proton 
resonance frequency chemical shift difference between brain-water and $N$-acetylaspartate have been reported and were used in this study. An adiabatically localized technique (semi-localization by adiabatic selective refocusing [sLASER]) coupled with an improved magnetic field shimming routine (gradient refocused echo shim) was implemented in all thermometry acquisitions due to its superior excitation and localization profiles, signal-to-noise, spectral quality, magnetic field homogeneity, and relative immunity to chemical shift displacement effects. ${ }^{31}$

\section{MR Spectroscopy Acquisition}

Multivoxel MR spectroscopy (MRSI) measurements were acquired for thermometry analysis. The T1-MPRAGE sequence was used for planning an MRSI protocol acquired with sLASER (TR = $1700 \mathrm{~ms}$; TE $=35 \mathrm{~ms}$; complex data points $=1024$; voxel acquisition grid $=16 \times 16$; voxel $\mathrm{ROI}=8 \times 8$; nominal voxel resolution $=5 \times 5 \times 12 \mathrm{~mm}^{3} ; 3-12$ averages for water-suppressed scans and 1 average for non-water-suppressed scans). A water suppression enhanced through $\mathrm{T} 1$ effects algorithm was used $(50-\mathrm{Hz}$ water suppression), and lipid saturation bands were placed outside the ROI. B0 shimming was achieved by using a gradient refocused echo shim algorithm ${ }^{35}$ with further refinement to the shim conditions achieved by secondary manual shimming. MRSI data were processed by using LCModel (http://www.lcmodel.com/). ${ }^{36}$ The non-water-suppressed scan was used for phase and eddy current corrections. Voxels with NAA Cramér-Rao lower bound values of $>20$ were excluded from analysis.

\section{MR Thermometry Analysis}

Voxelwise absolute temperature values were approximated from the chemical shift difference between the temperature-dependent water and temperature-independent NAA peaks by using the relationship of $-0.01 \mathrm{ppm} /{ }^{\circ} \mathrm{C} .{ }^{34,37}$ The water peaks in both the non-water-suppressed and water-suppressed scans were acquired at the same center frequency. The final temperature map was displayed as a weighted-average bicubic interpolation for visualization only. Voxelwise values were used in all statistical and descriptive analyses. For the temperature differences relative to the systemic temperature, the average rectal temperature recorded during the respective MRSI scan was subtracted from the absolute brain temperature calculated voxelwise.

The MRSI voxel positions recorded by the scanner are in the same patient-based coordinate system as the anatomic images. Using the affine matrix from the MRSI file header, we rotated the MRSI grid perpendicular to the $\mathrm{z}$-axis in the patient-based coordinate system, with the center of the patient-based coordinate system located at the center of the MRSI grid. The anatomic images were then resectioned parallel to the MRSI grid to a final resolution of $0.12 \times 0.12 \times 1 \mathrm{~mm}^{3}$ by using a nearest-neighbor interpolation so that corresponding anatomic image sections fully encompassed the MRSI plane, facilitating visualization of the MRSI grid and temperature maps. To classify MRSI voxels according to tissue state, the MRSI grid was overlaid onto diffusionweighted images and classified; by a single neuroradiologist experienced in stroke imaging (S.D.). All postprocessing was performed by using in-house-developed software written in Matlab 2015a (MathWorks, Natick, Massachusetts).

\section{Statistical Analysis}

Statistical analysis was performed in SPSS, Version 22.0 (IBM, Armonk, New York). Data are reported as mean and SD unless otherwise noted. Histograms are binned at $0.5^{\circ} \mathrm{C}$. To determine the relationship among stroke progression, infarct volume, and temperature, we used fixed- and mixed-effects linear models. The Schwarz-Bayesian information criterion was used to compare models and identify the presence of random, within-subject effects, including subject-dependent slopes and intercepts. For models with similar Schwarz-Bayesian information criterion values, the parsimonious model was used.

Infarct volume (cubic centimeters) on DWI at each time point was normalized to the final infarct volume at 24 hours. Time was treated as a continuous variable and reported in hours relative to the first MR spectroscopy measurement acquired 1 hour postsurgery. The change in temperature as a function of time and infarct volume was examined for systemic, ipsilateral brain, contralateral brain, and infarcted brain temperatures. For regressions of temperature as a function of time, temperature and tissue classification were used as a covariate in the model to examine differences between systemic and brain temperatures. Two-tailed $t$ tests were used to determine significance at $P<.05$. Multiple comparisons were adjusted post hoc by the Bonferroni correction.

\section{RESULTS}

Superselective, distal M2 occlusion was achieved in all 3 subjects. No operative or perioperative complications were encountered in 2 of the 3 experiments. Arterial extravasation was encountered due to traumatic arterial injury in an M3 branch of a single subject, with a short duration of extravasation before hemostasis was secured, producing trace subarachnoid hemorrhage isolated to the right parietal vertex. The postoperative course was unremarkable in this subject throughout the $\mathrm{t}^{0}$ and $\mathrm{t}^{1}$ follow-up, and satisfactory neuroimaging and thermometry were achieved across both imaging sessions.

Infarction volumes at the initial and final postoperative DWI scan are presented in Table 1, with the systemic (rectal), ipsilateral cerebral, and contralateral cerebral absolute temperature ranges in each primate throughout the $\mathrm{t}^{0}$ scanning session. In all 3 experiments, an initial systemic temperature drop was observed during $\mathrm{t}^{-7}, \mathrm{t}^{0}$, and $\mathrm{t}^{1}$ scanning sessions related to anesthesia induction, with steady systemic rewarming during the remainder of all 3 sessions in all 3 subjects. In preoperative scanning sessions, average ipsilateral, contralateral, and whole-brain temperatures remained coupled to the observed systemic temperature changes throughout the imaging session, while in contrast, all 3 infarction $\left(t^{0}\right)$ days exhibited progressive cerebral hyperthermia, significantly outpacing systemic temperature fluctuations and affecting both cerebral hemispheres (Figs 1 and 2).

\section{Relationship between Brain and Systemic Temperature}

A geographic distribution of temperatures at rest in all NHPs during $\mathrm{t}^{-7}$ scanning evolved to relatively widespread and progressive heating diffusely throughout the cerebral hemispheres as shown in the final $t^{0}$ time point (Fig 1). Similar trends were observed in all 3 stroke-induction experiments (Fig 2), in which 
Table 1: Subject-specific temperature and infarction volumes ${ }^{\mathrm{a}}$

\begin{tabular}{|c|c|c|c|}
\hline Parameter & Subject 1 & Subject 2 & Subject 3 \\
\hline \multicolumn{4}{|l|}{$\mathrm{T}^{-7}$ scan session } \\
\hline Systemic temperature range & $36.3-37.2$ & $36.8-37.9$ & $36.0-38.2$ \\
\hline Mean brain temperature range & $36.6 \pm 1.0-37.1 \pm 1.2$ & $36.7 \pm 0.6-37.8 \pm 1.1$ & $36.3 \pm 0.7-38.8 \pm 0.7$ \\
\hline Temperature difference: brain, systemic ${ }^{b}$ & $-0.1 \pm 1.2-0.2 \pm 1.0$ & $-0.1 \pm 0.6-0.4 \pm 1.1$ & $0.3 \pm 0.7-1.2 \pm 0.7$ \\
\hline \multicolumn{4}{|l|}{$\mathrm{T}^{\mathrm{O}}$ scan session } \\
\hline Infarct volume $1 \mathrm{hr}$ postsurgery $\left(\mathrm{cm}^{3}\right)$ & 0.42 & 0.28 & 6.70 \\
\hline Systemic temperature range & $36.7-37.6$ & $36.4-38.4$ & $35.7-38.8$ \\
\hline Mean ipsilateral brain temperature range & $37.4 \pm 1.1-40.7 \pm 1.4$ & $34.3 \pm 1.1-40.3 \pm 1.2$ & $35.9 \pm 0.6-41.6 \pm 2.9$ \\
\hline Mean contralateral brain temperature range & $37.4 \pm 0.8-40.4 \pm 0.7$ & $34.1 \pm 1.0-40.4 \pm 0.7$ & $35.8 \pm 0.5-42.2 \pm 1.8$ \\
\hline Temperature difference: ipsilateral brain, systemic & $0.7 \pm 1.1-3.2 \pm 1.4$ & $-2.1 \pm 1.1-2.5 \pm 1.3$ & $0.2 \pm 0.6-2.8 \pm 2.9$ \\
\hline Temperature difference: contralateral brain, systemic & $0.7 \pm 0.8-3.0 \pm 0.7$ & $-2.3 \pm 1.0-2.3 \pm 1.2$ & $0.1 \pm 0.5-3.4 \pm 1.8$ \\
\hline \multicolumn{4}{|l|}{$\mathrm{T}^{\top}$ scan session } \\
\hline Infarct volume $24 \mathrm{hr}$ postsurgery $\left(\mathrm{cm}^{3}\right)$ & 23.82 & 1.29 & 25.58 \\
\hline Systemic temperature & 35.6 & 35.1 & 34.6 \\
\hline Mean brain temperature & $37.9 \pm 1.0$ & $36.9 \pm 1.2$ & $36.3 \pm 0.9$ \\
\hline Temperature difference: brain, systemic & $2.3 \pm 1.0$ & $1.7 \pm 1.2$ & $1.7 \pm 0.9$ \\
\hline
\end{tabular}

${ }^{a}$ All temperatures are reported in degrees Celsius. Reported temperature ranges represent the full dynamic range from the indicated imaging session beginning from matched initial systemic and imaging temperature time points.

${ }^{b}$ Indicates a range of systemic-versus-brain temperature offsets.
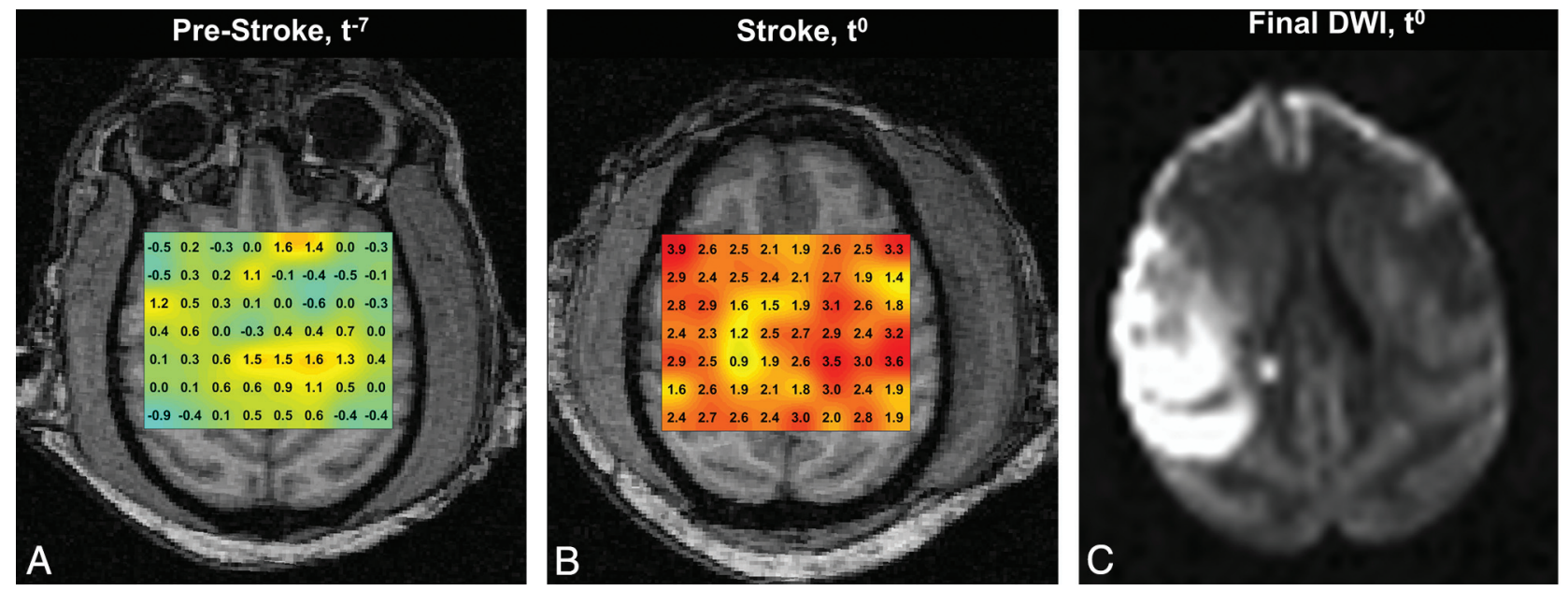

FIG 1. MR thermographs in subject 3 obtained during $\mathrm{t}^{-7}(A)$ and $\mathrm{t}^{0}(B)$ sessions, overlaid on T1-MPRAGE images. Terminal DWI (C) obtained at the conclusion of the $\mathrm{t}^{0}$ session following endovascular stroke induction demonstrates a large MCA territory infarction. Thermographs in $A$ and $B$ are presented in equivalent color scales, depicting voxelwise brain versus systemic temperature gradients. Disturbance to the geographic distribution of brain temperature gradients present during physiologic $t^{0}$ conditions are present, with notable, diffuse cerebral hyperthermia affecting both hemispheres in B. Generalized cerebral heating following infarction corresponds to significant elevation above decoupled systemic temperatures (not shown) as a function of time and infarction volume (see text).

matched $\mathrm{t}^{-7}$ and $\mathrm{t}^{0}$, systemic and cerebral temperatures are presented as a function of time. Coupled systemic and whole-brain temperature changes are shown during $\mathrm{t}^{-7}$ scanning, in which initially subphysiologic temperatures during early, postanesthesia scanning demonstrate slow increases of both systemic and brain temperatures in subsequent acquisitions under rewarming by the heating blanket. By comparison, following infarction, divergent brain and systemic temperatures are observed with time, despite slow development of febrile systemic temperature. Individual regressions of the normalized time from stroke onset (Fig 3) demonstrated highly significant associations for systemic temperature, as well as absolute (spectroscopic) ipsilateral hemispheric temperature, contralateral hemispheric temperature, and the temperature of DWI infarction core voxels (F-statistic, $P<.0005$ for all regressions). Comparison of the slopes of regression (Table 2) of systemic temperature, absolute brain temperature, ipsilateral brain temperature, and contralateral brain temperature with DWI infarction core confirmed significant overall differences ( $F$-statistic, $P=.007$ ), notably with significant differences between systemic temperatures and ipsilateral $(P=.007)$, contralateral $(P=.004)$, and infarction core $(P=.003)$ temperatures following post hoc multiplecomparison Bonferroni correction. A significant difference was not detected among hemispheric temperatures $(P>.05)$. Voxelwise analysis of the difference between brain and systemic temperatures in the ipsilateral-versus-contralateral hemisphere brain temperatures at the initial and final time points was characterized in a linear fixed-effects model, demonstrating no significant difference between hemispheres $(P>$ $.05)$.

The progressive divergence of systemic and brain temperatures between initial and final $t^{0}$ time points is illustrated histographically (Fig 4) for all 3 subjects, exhibiting increasing brain versus systemic temperature offsets with time. 

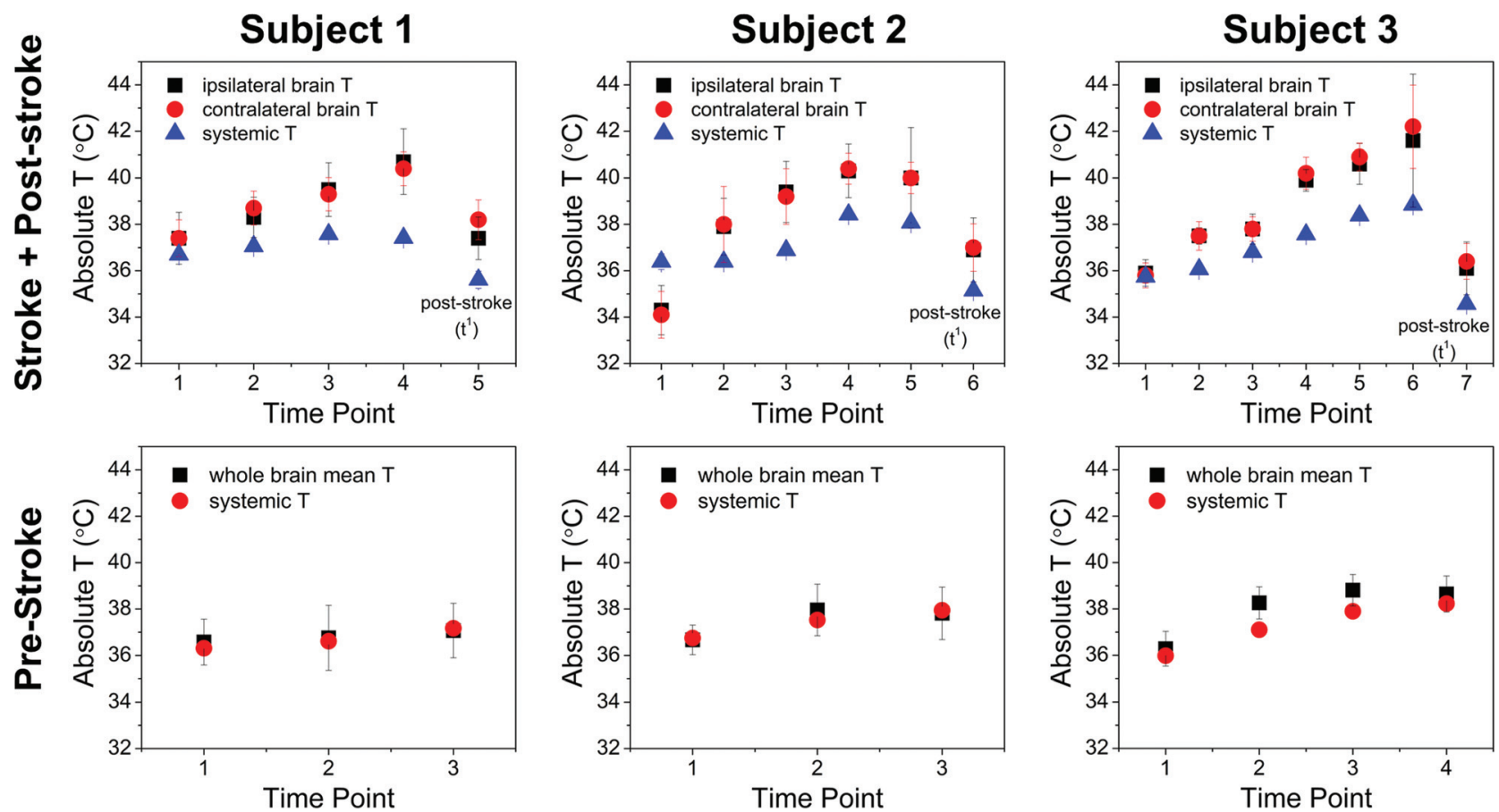

FIG 2. Subject-specific absolute temperature versus time for paired prestroke and stroke sessions. For each subject, the vertical columns represent $\mathrm{t}^{0}$ above and $\mathrm{t}^{-7}$ below. The $\mathrm{y}$-axis in $\mathrm{t}^{0}$ plots represents MR imaging-derived temperatures for the hemisphere ipsilateral and contralateral to the infarction, as well as systemic temperatures. All plots are presented in the same vertical scale, with errors bars (SD) as indicated. The final time point in all $t^{0}$ plots represents values from the poststroke session. Progressive heating of both cerebral hemispheres is present in all 3 subjects, outpacing the progressive systemic febrile temperatures in stroke aftermath. Brain hyperthermia is noted to resolve in the $\mathrm{t}^{1}$ session for all subjects. By comparison, all baseline $\mathrm{t}^{-7}$ scans exhibit closely coupled brain systemic temperatures, despite fluctuations related to early postanesthetic hypothermia during subject preparation.

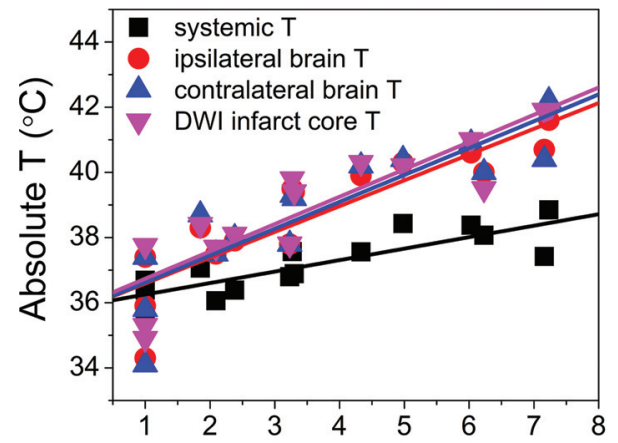

Normalized Time (hours post-infarct)

FIG 3. Aggregated fit from individual regressions from each subject, depicted for systemic temperatures, average hemispheric temperature ipsilateral and contralateral to infarction, and those voxels defined within the infarction territory. Individual regressions derived from a linear fixed-effects model demonstrate highly significant associations among all variables relative to time, as well as significant differences between brain temperatures and systemic temperatures following multiple-comparison correction (Table 2).

\section{Relationship between Infarction Volume and Temperature}

Individual regressions of normalized infarction volumes revealed significant associations with systemic $(P=.01)$ and ipsilateral brain $(P=.04)$ temperature but not with contralateral brain temperature $(P=.08)$. Importantly, a highly significant association was identified between normalized infarction volume and absolute infarction core temperatures $(P<.0005)$.

\section{DISCUSSION}

These findings represent, to our knowledge, the first such demonstration of dynamic ischemic brain temperature monitoring by using fully noninvasive MR thermometry. With a controlled NHP stroke-induction model, the findings confirmed: 1) the presence of physiologic brain temperature gradients in the healthy brain, 2) disturbance of brain temperatures following ischemia, and 3) decoupling of baseline brain systemic temperature gradients as hypothesized, in a phylogenetically similar substrate offering potential insight into human spatiotemporal brain temperature disturbance.

Febrile systemic temperatures are a well-known consequence of large-vessel-occlusion stroke, particularly in the later acute phase of ischemia; however, the immediacy with which brain temperatures increased in our study suggests that damaging brain temperature elevations may indeed begin early following infarction. Cerebral temperatures correlated strongly with infarction volumes in this study, and while a causal relationship could not be established in our experimental design, the findings are consistent with existing reports of infarction expansion and poor outcome among fully revascularized patients with febrile stroke. ${ }^{8,10,11,13,26,38,39}$ Brain temperature regulation is expected during systemic febrile responses; however, these findings support the notion that brain injury may attenuate inherent brain thermoregulatory mechanisms..$^{7,11,18,37,40}$ More surprising perhaps is the rapid decoupling of brain systemic temperatures as observed in this study, whereby brain temperatures steadily outpaced even the increasing systemic temperatures. Several potential explanations can be considered, which at present remain dif- 
Table 2: Absolute systemic and cerebral temperatures versus time ${ }^{\mathrm{a}}$

\begin{tabular}{lcccccc}
$\begin{array}{c}\text { Temperature } \\
\text { Parameter }\end{array}$ & Slope $\left({ }^{\circ} \mathrm{C} / \mathrm{hr}\right)$ & Intercept $\left({ }^{\circ} \mathrm{C}\right)$ & $\begin{array}{c}\text { F-Stat of } \\
\text { Regression }(\boldsymbol{d f})\end{array}$ & $\begin{array}{c}\text { P Value } \\
(\boldsymbol{F} \text {-Stat) }\end{array}$ & $\begin{array}{c}\text { Difference in } \\
\text { Slope versus Systemic } \\
\text { Temperature }\end{array}$ & $\begin{array}{c}\boldsymbol{P} \text { Value of } \\
\text { Difference versus } \\
\text { Systemic }\end{array}$ \\
\hline Systemic & $0.352(0.062)$ & $35.9(0.3)$ & $32.2(1,13)$ & $<.0005^{\mathrm{b}}$ & $\mathrm{NA}$ & $\mathrm{NA}$ \\
Ipsilateral brain & $0.791(0.116)$ & $35.8(0.5)$ & $47.0(1,13)$ & $<.0005^{\mathrm{b}}$ & $0.439(0.156)$ & $.007^{\mathrm{b}}$ \\
Contralateral brain & $0.824(0.129)$ & $35.8(0.5)$ & $40.7(1,13)$ & $<.0005^{\mathrm{b}}$ & $0.471(0.156)$ & $.004^{\mathrm{b}}$ \\
DWI infarct core & $0.838(0.122)$ & $35.9(0.5)$ & $47.5(1,13)$ & $<.0005^{\mathrm{b}}$ & $0.486(0.156)$ & $.003^{\mathrm{b}}$ \\
\hline
\end{tabular}

Note:-F-Stat indicates F-statistic; NA, not applicable.

a Parameter estimates (standard error when applicable) from a linear fixed-effects model reported relative to normalized time since stroke onset (defined at initiation of scanning 1 hour following stroke induction).

${ }^{\mathrm{b}}$ Indicates statistical significance at $P<.05$, results of 2 -tailed $t$ test of $F$-statistics.

\section{Subject 1}
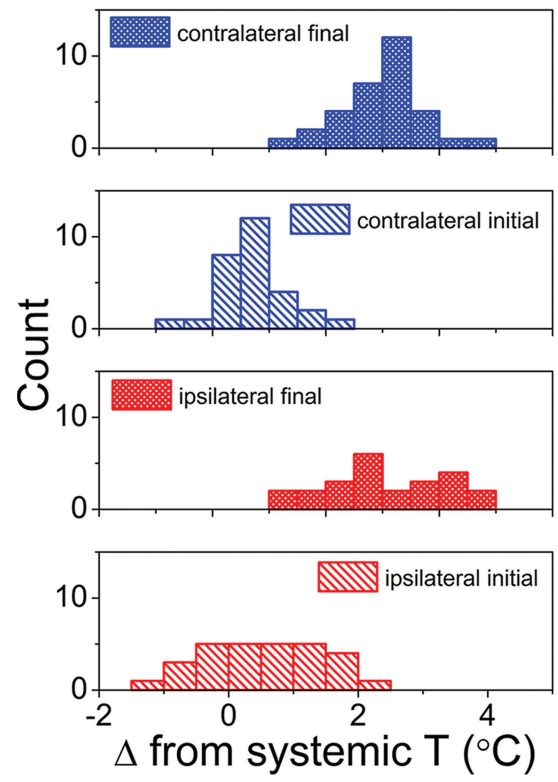

Subject 2
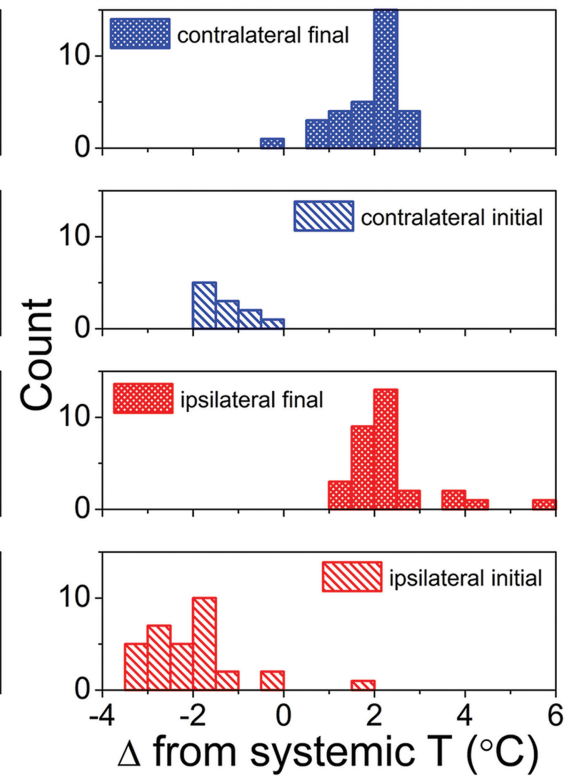

Subject 3
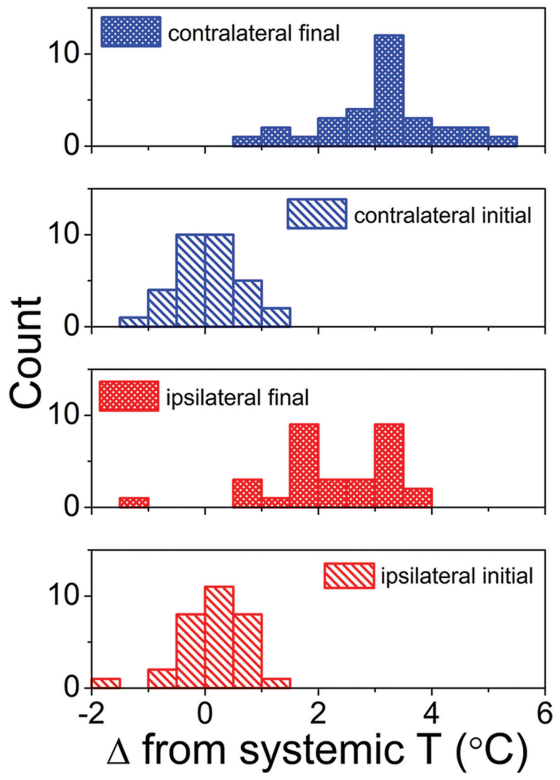

FIG 4. Subject-specific histograms of hemispheric cerebral temperature versus time reflecting divergence of systemic and brain temperatures. The $x$-axis represents the brain-systemic temperature offset, represented respectively for the initial and final time points, both for ipsilateral and contralateral brain temperatures. The rightward shift of all histograms reflects progressive cerebral hyperthermia decoupled from systemic temperatures present for both hemispheres of all 3 subjects.

ficult to conclusively establish: First, cerebral temperatures may theoretically surpass systemic temperatures in hypoperfused-butviable tissues (ie, the putative ischemic penumbra), where metabolically active but ischemic tissues are perfused insufficiently to ensure ready clearance of metabolic heat. ${ }^{7,37,41,42}$ This, by comparison to more mature areas of infarction among which hypoperfusion of metabolically inert tissues may be inconsequential with respect to temperature in the acute phase before inflammatory cascades initiate. This explanation seems unlikely, at least in isolation, given that such changes would be expected locally or regionally, rather than globally affecting both hemispheres as observed in our study. The relation among temperature, viability, and perfusion could not be tested rigorously in this study, as discussed further among the limitations below, but nevertheless the impact is likely only partial.

A second consideration may be that of heating influences on cerebral tissues related to inflammatory pathways at play following infarction. Recent investigations have emphasized the immunologic basis of stroke progression, underscoring numerous effectors of stroke progression. ${ }^{43,44}$ Importantly, these temperature changes occur independent of infection and appear to be driven by pyrogenic cytokines or lymphocyte trafficking. ${ }^{9}$ These increasing temperatures may, in turn, beget further injury, driving progression of infarction and degrading the temperature-sensitive blood-brain barrier, inciting a continued cycle of injury. Whether immunologic mechanisms can be imputed in these findings remains to be studied but could represent a treatment target, perhaps allowing neuroprotection, together with therapeutic hypothermia, as proposed in other settings. Last, tissue swelling developing with ischemia could compete with heat dissipation to the brain surface; however, heat conduction is primarily believed to modulate surface brain temperatures and is therefore likely a less important variable in comparison with hemodynamic, metabolic, or immunologic factors. $5,21,22,24$

While simulations and biophysical models of brain temperature regulation have been proposed, validation by direct in vivo observations is limited to a relatively small number of human and nonhuman animal studies. ${ }^{2,3,5,17,21,24,26,28,45-48}$ Prevailing paradigms highlight the complexity of brain temperature as a biomarker, reflecting the intersection of complex metabolic and hemodynamic pathways. ${ }^{5,22}$ Accordingly, brain temperature may, to some extent, be viewed as an epiphenomenon to heat-producing 
metabolic pathways (primarily the cleavage/use of oxygen delivered by the heme moiety within circulating red blood cells) and heat-dissipating mechanisms (consisting principally of circulating blood flow) critical to elimination of thermal waste through countercurrent heat-sink mechanisms, which depend on brain systemic temperature gradients. ${ }^{1,3,17,49}$ Thus, temperature may be an indirect product of brain metabolic homeostasis, albeit directly affected by hypothalamic or other controls, including proximity to the brain surface, CSF spaces, and pneumatized cells of the paranasal sinuses. The development of refined systems to protect the brain from dangerous heating, such as during systemic hyperthermia, may have paralleled loss of the rete caroticum in humans and NHPs. ${ }^{1,3,17,49}$ Critically, however, the injured brain may lose this thermoregulatory capacity, allowing brain heating under systemic or even local-regional influences.

Practical considerations have historically challenged the study of brain temperature regulation and the development of a generalizable formalism, due to the absence of safe, direct brain thermometry techniques. ${ }^{3,17,26,45,46,50}$ Generally limited to invasive and costly implantable probes and thermocouples, the spatial distribution of temperature has remained relatively unaddressed. Past studies have suggested that brain temperature, at least centrally, exceeds systemic temperatures due to its high metabolic rate, consuming $20 \%$ of oxygen and $25 \%$ of glucose at rest, yet accounting for only $2 \%-3 \%$ of body weight. $5,9,24,48$ The use of noninvasive, temperature-sensitive nuclear magnetic resonance phenomena has been proposed for this purpose, and a variety of potential methodologies exist within the current armamentarium. ${ }^{28,33,34,42,51}$ MRSI proves particularly advantageous because identification of an internally referenced chemical shift difference such as that between brain-water and $\mathrm{N}$-acetylaspartate permits nearly absolute thermometry, critical for thermograph production across imaging sessions and facilitating intra- and intersubject temperature characterization, by comparison with faster but purely relative phase-contrast thermometry. ${ }^{33,34}$ A previous phantom-optimization study tested 3 distinct analysis methods, all of which performed extremely well compared with real-time fiber-optic temperature monitoring $\left(R^{2}>99\right.$, root-mean-square deviation $\sim 0.005, P<.001$ ), and we proposed an extension of this approach for multiphasic, multivoxel NHP brain thermometry in the current study. The findings in the present study differ in important ways from those in past investigations, including recent reports by Karaszewski et $\mathrm{al}^{7}$ of noninvasive brain thermometry following acute ischemic stroke in humans. Specifically in their study, initial MR imaging may have been delayed by $>24$ hours from the time of onset and not repeated until 3-7 days following presentation. Similarly, baseline temperatures before stroke onset critically inform the magnitude and direction of temperature disturbance but cannot be reasonably obtained outside the setting of controlled stroke induction.

The Rhesus Macaque brain provides a robust prehuman experimental model in stroke neuroscience, in which rodent and lower primate protocols may fail translation to humans. ${ }^{52}$ MRSI in the Rhesus brain, however, poses certain challenges. The exuberant cranial fat may significantly degrade spectral quality, despite meticulous outer volume suppression or fat-water separation strategies. To address these limitations, an optimized approach combines sLASER spectroscopy with improved shimming routines (gradient refocused echo shim) to better offset commonly suboptimal shim conditions near the brain surface. ${ }^{31,53}$ In doing so, superior MR spectroscopy thermometry can be achieved, with higher spectral quality, greater SNR, longer metabolite $\mathrm{T} 2{ }^{*}$, and amelioration of chemical shift displacement artifacts corrupting conventional approaches to MRSI.

We acknowledge several study limitations, including primarily the sample size. We sought to characterize brain temperatures under physiologic and controlled ischemic conditions, with sufficiently high temporal sampling, to inform a basic paradigm of spatiotemporal temperature evolution and brain-systemic temperature coupling. Experimental demands of sourcing NHPs to conduct 12-15 hours of MR imaging during 7-10 days of animal care and to undertake stroke induction prohibited greater recruitment. Nevertheless, recent expert consensus has emphasized the importance of stroke neuroscience research models in gyrencephalic primates to bolster translational research designs and facilitate applicability to human physiology and disease. ${ }^{52,54}$ The invasive study of NHPs more importantly presents ethical considerations demanding thoughtful study design, and to this end, the present study was guided by contemporary philosophies on the humane treatment of study subjects to eliminate or minimize pain, distress, anxiety, and depression. ${ }^{52}$

Similar considerations precluded the addition of a control population of nonischemic or sham-operated NHPs, and potential bias in this respect cannot be excluded. The experimental design furthermore limited our ability to acquire reliable, contemporaneous perfusion data in study subjects. Certainly, cerebral perfusion would represent a valuable adjunctive parameter in this setting; however, hardware limitations precluded acquisition of arterial spin-labeling perfusion by using the same transmit-receive coil system tuned and configured for optimized NHP brain imaging and spectroscopy. A 2-coil arterial spin-labeling solution was not achievable with our experimental resources, and multiphasic dynamic susceptibility contrast perfusion could not be performed due to unrecoverable degradation of proton resonance spectra for accurate thermometry. Further study of the relation among perfusion, temperature, and viability, ideally coupled to oxygen metabolism, are warranted and the subject of investigation in our laboratory.

Despite the absence of perfusion imaging, the present study offers valuable insight into cerebral thermoregulation and dysregulation in a biologically relevant model of acute ischemic stroke. The use of the chemical shift difference between brainwater and $N$-acetylaspartate could theoretically be impacted by diminishing NAA concentrations in injured tissues, precluding identification of resonance signatures and therefore thermometry; however, in agreement with earlier studies by Corbett et al, ${ }^{27}$ NAA concentrations throughout the early aftermath of stroke remained sufficient to permit thermometry, though a more extended survival study beyond the acute stage could potentially require the use of another non-hydrogen-bound proton metabolite as previously described.

Last, the impact of anesthesia induction must be addressed. General anesthesia may significantly affect brain or body temperatures; however, this pharmacologic profile played favorably into 
our design, in which all 3 baseline scans, stroke sessions, and poststroke day scans shared a common induction protocol. This feature proved valuable as an additional form of physiologic contrast, allowing, first, for characterization of brain systemic gradients during the initial cooling phase following induction, at which time the subject was exposed to cool ambient temperatures. Thereafter, during systemic warming by the circulating water blanket, brain and systemic (rectal) temperatures could be monitored simultaneously. Cooling maneuvers were not prescribed in our protocol, and when subject temperatures began to exceed physiologic temperatures set by the water bath, as they invariably did under the heating blanket, the water bath automatically powered down and the blanket was removed.

Significant systemic temperature elevations were not specifically noted among the 3 examination sessions, perhaps due to the very early postinfarction monitoring period, in comparison with the slightly more delayed fevers described in recent stroke studies. However, of interest to our understanding of these mechanisms is that the cerebral-systemic gradient changed only minimally during physiologic baseline scanning, despite clear changes to each independently, following anesthesia induction and subsequent rewarming. In contrast, progressive uncoupling of brain and systemic temperatures was consistently observed after stroke, despite escalations in both, consistent with existing hypotheses that brain injury impairs cerebral thermoregulation independent of systemic temperatures. The strongest evidence for such decoupling may come from poststroke $\left(\mathrm{t}^{1}\right)$ scans, in which even early postanesthesia thermographs during diminished systemic temperatures revealed elevated brain systemic gradients before systemic rewarming by the heating blanket could be achieved as shown in Table 1. This finding may suggest that therapeutic targets for brain cooling may be necessary, even in advance of the development of systemic febrile responses, often delayed for days following stroke.

\section{CONCLUSIONS}

These findings further establish the feasibility of noninvasive thermometry during ischemic injury and support theorized disturbances in cerebral thermoregulation following acute ischemic stroke.

Disclosures: Seena Dehkharghani-RELATED: Grant: American Society of Neuroradiology Scholar Award in Neuroradiology Research. Candace C. FleischerUNRELATED: Grants/Grants Pending: National Institutes of Health F32 individual postdoctoral fellow, Comments: salary support from a National Institutes of Health postdoctoral fellowship for work unrelated to this project; Travel/Accommodations/ Meeting Expenses Unrelated to Activities Listed: International Society for Magnetic Resonance in Medicine and American Chemical Society, Comments: trainee stipends for the International Society for Magnetic Resonance in Medicine annual meeting (Singapore, Toronto) and the International Society for Magnetic Resonance in Medicine MRS workshop (Germany), travel award to the American Chemical Society Postdoc to Faculty workshop. Deqiang Qiu-UNRELATED: Grants/Grants Pending: Siemens Medical Solutions.* Frank Tong—RELATED: Grant: American Society of Neuroradiology Research Grant. * *Money paid to the institution.

\section{REFERENCES}

1. Baker MA. A brain-cooling system in mammals. Sci Am 1979;240: 130-39 CrossRef Medline

2. Brengelmann GL. Specialized brain cooling in humans? FASEB J 1993;7:1148-52; discussion 1152-53 Medline

3. Cabanac M. Selective brain cooling in humans: "fancy" or fact? FASEB J 1993;7:1143-46; discussion 1146-47 Medline

4. Hayward JN, Baker MA. Role of cerebral arterial blood in the regu- lation of brain temperature in the monkey. Am J Physiol 1968;215: 389-403 Medline

5. Sukstanskii AL, Yablonskiy DA. Theoretical model of temperature regulation in the brain during changes in functional activity. Proc Natl Acad Sci U S A 2006;103:12144-49 CrossRef Medline

6. Busto R, Dietrich WD, Globus MY, et al. Small differences in intraischemic brain temperature critically determine the extent of ischemic neuronal injury. J Cereb Blood Flow Metab 1987;7:729-38 CrossRef Medline

7. Karaszewski B, Carpenter TK, Thomas RG, et al. Relationships between brain and body temperature, clinical and imaging outcomes after ischemic stroke. J Cereb Blood Flow Metab 2013;33:1083-89 CrossRef Medline

8. Kil HY, Zhang J, Piantadosi CA. Brain temperature alters hydroxyl radical production during cerebral ischemia/reperfusion in rats. J Cereb Blood Flow Metab 1996;16:100-06 Medline

9. Axelrod YK, Diringer MN. Temperature management in acute neurologic disorders. Neurol Clin 2008;26:585-603, xi CrossRef Medline

10. Dehkharghani S, Bowen M, Haussen DC, et al. Body temperature modulates infarction growth following endovascular reperfusion. AJNR Am J Neuroradiol 2016 Oct 6. [Epub ahead of print] Medline

11. Campos F, Blanco M, Barral D, et al. Influence of temperature on ischemic brain: basic and clinical principles. Neurochem Int 2012; 60:495-505 CrossRef Medline

12. Otawara Y, Ogasawara K, Kubo Y, et al. Brain and systemic temperature in patients with severe subarachnoid hemorrhage. Surg Neurol 2003;60:159-64; discussion 164 CrossRef Medline

13. Reith J, Jørgensen HS, Pedersen PM, et al. Body temperature in acute stroke: relation to stroke severity, infarct size, mortality, and outcome. Lancet 1996;347:422-25 CrossRef Medline

14. Sun Z, Zhang J, Chen Y, et al. Differential temporal evolution patterns in brain temperature in different ischemic tissues in a monkey model of middle cerebral artery occlusion. J Biomed Biotechnol 2012; 2012:980961 CrossRef Medline

15. Verlooy J, Heytens L, Veeckmans G, et al. Intracerebral temperature monitoring in severely head injured patients. Acta Neurochir (Wien) 1995;134:76-78 CrossRef Medline

16. Wang CX, Stroink A, Casto JM, et al. Hyperthermia exacerbates ischaemic brain injury. Int J Stroke 2009;4:274-84 CrossRef Medline

17. Cabanac M, Caputa M. Natural selective cooling of the human brain: evidence of its occurrence and magnitude. J Physiol 1979;286: 255-64 CrossRef Medline

18. Caputa M. Selective brain cooling: a multiple regulatory mechanism. J Therm Biol 2004;29:691-702 CrossRef

19. Kalmbach AS, Waters J. Brain surface temperature under a craniotomy. J Neurophysiol 2012;108:3138-46 CrossRef Medline

20. Kuhnen G, Jessen C. Thermal signals in control of selective brain cooling. Am J Physiol 1994;267:R355-59 Medline

21. Nelson DA, Nunneley SA. Brain temperature and limits on transcranial cooling in humans: quantitative modeling results. Eur J Appl Physiol Occup Physiol 1998;78:353-59 CrossRef Medline

22. Yablonskiy DA, Ackerman JJ, Raichle ME. Coupling between changes in human brain temperature and oxidative metabolism during prolonged visual stimulation. Proc Natl Acad Sci US A 2000; 97:7603-08 CrossRef Medline

23. Zenker W, Kubik S. Brain cooling in humans-anatomical considerations. Anat Embryol (Berl) 1996;193:1-13 Medline

24. Zhu M, Ackerman JJ, Sukstanskii AL, et al. How the body controls brain temperature: the temperature shielding effect of cerebral blood flow. J Appl Physiol (1985) 2006;101:1481-88 Medline

25. Simon E. Tympanic temperature is not suited to indicate selective brain cooling in humans: a re-evaluation of the thermophysiological basics. Eur J Appl Physiol 2007;101:19-30 Medline

26. Schwab S, Spranger M, Aschoff A, et al. Brain temperature monitoring and modulation in patients with severe MCA infarction. $\mathrm{Neu}$ rology 1997;48:762-67 Medline

27. Corbett RJ, Purdy PD, Laptook AR, et al. Noninvasive measurement 
of brain temperature after stroke. AJNR Am J Neuroradiol 1999;20: 1851-57 Medline

28. Corbett RJ, Laptook AR, Tollefsbol G, et al. Validation of a noninvasive method to measure brain temperature in vivo using $1 \mathrm{H}$ NMR spectroscopy. J Neurochem 1995;64:1224-30 Medline

29. Corbett R, Laptook A, Weatherall P. Noninvasive measurements of human brain temperature using volume-localized proton magnetic resonance spectroscopy. J Cereb Blood Flow Metab 1997;17:363-69 Medline

30. Dehkharghani S, Mao H, Howell L, et al. Proton resonance frequency chemical shift thermometry: experimental design and validation toward high-resolution noninvasive temperature monitoring and in vivo experience in a nonhuman primate model of acute ischemic stroke. AJNR Am J Neuroradiol 2015;36:1128-35 CrossRef Medline

31. Dehkharghani S, Wei L, Mao H, et al. Multivoxel proton spectroscopy for non-invasive MR thermometry: phantom comparison of PRESS and semiLASER-localized chemical shift imaging for temperature monitoring. In: Proceedings of the Annual Meeting of the International Society for Magnetic Resonance in Medicine and the European Society for Magnetic Resonance in Medicine, Milan, Italy. May $10-16,2014: 2828$

32. Tong FC, Zhang X, Kempf DJ, et al. An enhanced model of middle cerebral artery occlusion in nonhuman primates using an endovascular trapping technique. AJNR Am J Neuroradiol 2015;36:2354-59 CrossRef Medline

33. Rieke V, Butts Pauly K. MR thermometry. J Magn Reson Imaging 2008;27:376-90 Medline

34. Kuroda K. Non-invasive MR thermography using the water proton chemical shift. Int J Hyperthermia 2005;21:547-60 Medline

35. Zhong X, Lyubich YM, DeVito T, et al. Quantitative comparison of shim algorithms for in vivo 1H-MRS. In: Proceedings of the Annual Meeting of the International Society for Magnetic Resonance in Medicine, Melbourne, Australia. May 5-11, 2012:4397

36. Provencher SW. Automatic quantitation of localized in vivo $1 \mathbf{H}$ spectra with LCModel. NMR Biomed 2001;14:260-64 Medline

37. Marshall I, Karaszewski B, Wardlaw JM, et al. Measurement of regional brain temperature using proton spectroscopic imaging: validation and application to acute ischemic stroke. Magn Reson Imaging 2006;24:699-706 Medline

38. Kammersgaard LP, Jørgensen HS, Rungby JA, et al. Admission body temperature predicts long-term mortality after acute stroke: the Copenhagen Stroke Study. Stroke 2002;33:1759-62 Medline

39. Wolfe KB. Effect of hypothermia on cerebral damage resulting from cardiac arrest. Am J Cardiol 1960;6:809-12 Medline

40. Karaszewski B, Wardlaw JM, Marshall I, et al. Early brain temperature elevation and anaerobic metabolism in human acute ischaemic stroke. Brain 2009;132:955-64 Medline

41. Derdeyn CP, Videen TO, Fritsch SM, et al. Compensatory mecha- nisms for chronic cerebral hypoperfusion in patients with carotid occlusion. Stroke 1999;30:1019-24 Medline

42. Karaszewski B, Wardlaw JM, Marshall I, et al. Measurement of brain temperature with magnetic resonance spectroscopy in acute ischemic stroke. Ann Neurol 2006;60:438-46 Medline

43. Chamorro A, Meisel A, Planas AM, et al. The immunology of acute stroke. Nat Rev Neurol 2012;8:401-10 CrossRef Medline

44. Gauberti M, Montagne A, Marcos-Contreras OA, et al. Ultra-sensitive molecular MRI of vascular cell adhesion molecule-1 reveals a dynamic inflammatory penumbra after strokes. Stroke 2013;44: 1988-96 CrossRef Medline

45. Mellergård $P$. Changes in human intracerebral temperature in response to different methods of brain cooling. Neurosurgery 1992;31: 671-77; discussion 677 Medline

46. Mellergård P, Nordström CH, Messeter K. Human brain temperature during anesthesia for intracranial operations. J Neurosurg Anesthesiol 1992;4:85-91 Medline

47. Soukup J, Rieger A, Holz C, et al. Temperature gradient between brain tissue and arterial blood mirrors the flow-metabolism relationship in uninjured brain: an experimental study. Acta Anaesthesiol Scand 2007;51:872-79 Medline

48. Sukstanskii AL, Yablonskiy DA. Theoretical limits on brain cooling by external head cooling devices. Eur J Appl Physiol 2007;101:41-49 Medline

49. Falk D. Brain evolution in Homo: the "radiator" theory. Behav Brain Sci 1990;13:333-44

50. Stone JG, Young WL, Smith CR, et al. Do standard monitoring sites reflect true brain temperature when profound hypothermia is rapidly induced and reversed? Anesthesiology 1995;82:344-51 Medline

51. Cady EB, D'Souza PC, Penrice J, et al. The estimation of local brain temperature by in vivo $1 \mathrm{H}$ magnetic resonance spectroscopy. Magn Reson Med 1995;33:862-67 Medline

52. Cook DJ, Tymianski M. Nonhuman primate models of stroke for translational neuroprotection research. Neurotherapeutics 2012;9: 371-79 CrossRef Medline

53. Fleischer CC, Qiu D, Zhong X, et al. Multivoxel proton magnetic resonance spectroscopy for non-invasive thermometry: improvements in spectra quality using semiLASER with GRE shim. In: Proceedings of the Annual Meeting of the International Society for Magnetic Resonance in Medicine and the European Society for Magnetic Resonance in Medicine, Toronto, Ontario, Canada. May 30 to June 5, 2015:4062

54. Stroke Therapy Academic Industry Roundtable (STAIR). Recommendations for standards regarding preclinical neuroprotective and restorative drug development. Stroke 1999;30:2752-58 Medline 